\title{
Change in mammography screening attendance after removing the out-of-pocket fee: a population-based study in Sweden (2014-2018)
}

\author{
Magdalena Lagerlund ${ }^{1}$ (D) Anna Åkesson ${ }^{2} \cdot$ Sophia Zackrisson ${ }^{1}$
}

Received: 4 December 2020 / Accepted: 2 July 2021 / Published online: 28 July 2021

(c) The Author(s) 2021

\begin{abstract}
Purpose To assess the change in mammography screening attendance in Sweden—overall and in sociodemographic groups at risk of low attendance-after removal of the out-of-pocket fee in 2016.

Methods Individual-level data on all screening invitations and attendance between 2014 and 2018 were linked to sociodemographic data from Statistics Sweden. Odds ratios and 95\% confidence intervals (CIs) for attendance by time period and sociodemographic factor were computed using mixed logistic regression to account for repeated measures within women. The study sample included 1.4 million women, aged 40-75, who had a mammography screening appointment in 2014-2015 and/or 2017-2018 in 14 of Sweden's 21 health care regions.

Results Overall screening attendance was $83.8 \%$ in $2014-2015$ and $84.1 \%$ in $2017-2018$ (+ 0.3 percentage points, $95 \%$ CI $0.2-0.4)$. The greatest increase in attendance was observed in non-Nordic women with the lowest income, where attendance rose from 62.9 to $65.8 \%$ ( +2.9 points, $95 \%$ CI $2.3-3.6$ ), and among women with four or more risk factors for low attendance, where attendance rose from 59.2 to $62.0 \%$ ( +2.8 points, $95 \%$ CI $2.2-3.4)$.

Conclusion Screening attendance did not undergo any important increase after implementing free screening, although attendance among some sociodemographic groups increased by almost three percentage points after the policy change.
\end{abstract}

Keywords Mammography $\cdot$ Breast cancer screening $\cdot$ Women's health $\cdot$ Socioeconomic aspects of health

\section{Abbreviations \\ OR Odds ratio \\ CI Confidence interval \\ SEK Swedish kronor (currency)}

\section{Introduction}

Most European countries have national population-based programs that offer mammography screening to women in varying age ranges between 40 and 74 [1]. Since the public health impact of population-based screening depends on

Magdalena Lagerlund

magdalena.lagerlund.1573@med.lu.se

1 Department of Translational Medicine, Diagnostic Radiology, Skåne University Hospital, Lund University, Malmö, Sweden

2 Clinical Studies Sweden - Forum South, Skåne University Hospital, Lund, Sweden high attendance in order to reduce breast cancer mortality, monitoring, as well as understanding and considering the factors influencing attendance, is important. In the European guidelines for quality assurance in breast cancer screening and diagnosis, attendance is listed as one of the key performance indicators where $>70 \%$ is stated as the acceptable level and $>75 \%$ as the desirable level [2].

Sweden has offered a nationwide outreach mammography screening program since 1997 [3, 4]. Overall attendance is typically about $80 \%$ [1], but is lower in groups who may be socioeconomically vulnerable, such as women who were born abroad [5-8], have a low income [6-8], are unmarried or living without a partner [5, 7-10], are not gainfully employed [5-7, 10], or who have a lower education [5].

As part of the Swedish government's efforts to improve health care equality and women's health, mammography screening became free of charge on July 12016 [11], before which time most regions charged a small out-of-pocket fee ( $\leq 200 \mathrm{SEK} \approx \$ 23 \mathrm{USD}$ ). According to research conducted in the USA, reducing or removing the out-of-pocket fee can increase screening attendance [12-14]. However, a Swedish 
study found no correlation between fees $(0-170$ SEK $\approx$ \$0-20 USD) and attendance (66-91\%) in different regions in Sweden in 1995-1996 [4]. In a more recent study conducted in Stockholm County (Sweden), attendance rose from 68 to $70 \%$ after the screening fee was removed in 2012 [15].

The objective of this study was to assess the change in mammography screening attendance in Sweden-overall and in sociodemographic groups at risk of low attendanceafter removal of the out-of-pocket screening fee in 2016. We examined the change in attendance from 2014-2015 to 2017-2018, stratified by region and sociodemographic factors.

\section{Materials and methods}

This longitudinal population-based register study was conducted in Sweden, where women between the ages of 40 and 74 are invited to mammography screening every 18-24 months depending on age and regional capacity. All invitations are sent by post and offer a pre-booked appointment date and time, which does not need to be confirmed and can be rescheduled or canceled. Since each health care region individually conducts and administers their screening, there are differences in intervals between screening appointments, the layout and content of the invitation letter, hours of operation, ways of canceling or rescheduling appointments, reminders, etc. All but two (Stockholm and Östergötland) of the 21 health care regions charged a small out-of-pocket fee between 80 and 200 SEK ( $\approx \$ 9-23$ USD) before the implementation of free screening in 2016.

A study period between 2014 and 2018 was chosen to study the change in screening attendance during the two-year period before and after removal of the out-of-pocket fee in 2016. Individual screening-related data were extracted for all women invited to the screening program in 15 of 21 health care regions in Sweden. These regions used the same company (Sectra $A B$ ) for their radiological information system (RIS) to administer and track invitations, attendance, and results throughout the entire study period, which enabled high quality and consistency of data between regions. Of the six regions initially excluded, four (Jönköping, Kronoberg, Norrbotten, and Uppsala) used different radiological information systems for all or part of the study period, and two (Sörmland and Östergötland) did not grant us permission to extract data. Two of three programs operating in the Stockholm region granted permission. However, these were excluded from the final study sample, since they had already removed the out-of-pocket fee before the study period. This study was approved by the local ethics committee at Lund University (Nos. 2018/576 and 2018/965). Active informed consent as a requirement for data collection was waived.
The extracted data included the screening appointment date, age at the screening appointment, and attendance outcome (attended, canceled, missed, and unavailable), for each regional mammography program separately, and were combined into one dataset. The unique personal identity number assigned to every resident in Sweden was used to merge screening data with information on individual-level sociodemographic characteristics obtained from population registers at Statistics Sweden (the Longitudinal Integration Database for Health Insurance and Labour Market Studies (LISA) [16], the Total Population Register [17], and the Geodatabase [18]). To secure anonymity, Statistics Sweden replaced this number with an arbitrary code before releasing the data to the research group. The most recent sociodemographic information was used for each screening appointment. Sameyear sociodemographic data were linked to each screening appointment in 2014, 2015, and 2017; in 2018, same-year data were available only for home ownership and type of municipality, and data on income, education, and cohabitation from 2017 were used.

Initially, the dataset included a total of 4,582,477 appointments among 1,780,164 women in 15 regions (including Stockholm). The flow chart in Fig. 1 describes the different steps of exclusion, resulting in a final selection of $2,381,142$ appointments among 1,350,654 women in 14 regions (Fig. 2). These 14 regions encompass about $59 \%$ of the women eligible for mammography screening in Sweden and about $81 \%$ of the women affected by the fee removal. The most recent screening appointment for each woman was selected, aged 40-75, within each time period (2014-2015 and 2017-2018), excluding appointments during the transition year of 2016. Women 75 years of age were included to allow for overflow from the age limit of 74 years due to administrative reasons, e.g., rescheduling. Appointments were excluded when personal identity numbers lacked a match, had duplicates, or were suspected to have been recycled according to data from Statistics Sweden. Furthermore, appointments with examination or cancelation codes that were not related to mammography screening were excluded. Duplicate appointments within the same program (both identical and non-identical) at different locations and within the same year were excluded as well.

\section{Outcome variable}

The outcome variable in this study was mammography screening attendance (yes/no), irrespective of whether it was the original or a rescheduled appointment date, according to the most recent screening appointment for each woman during the periods 2014-2015 and 2017-2018. The rationale for studying two-year time periods was to allow for longer screening cycles, which is common in several regions. 
Fig. 1 Selection of the final study sample

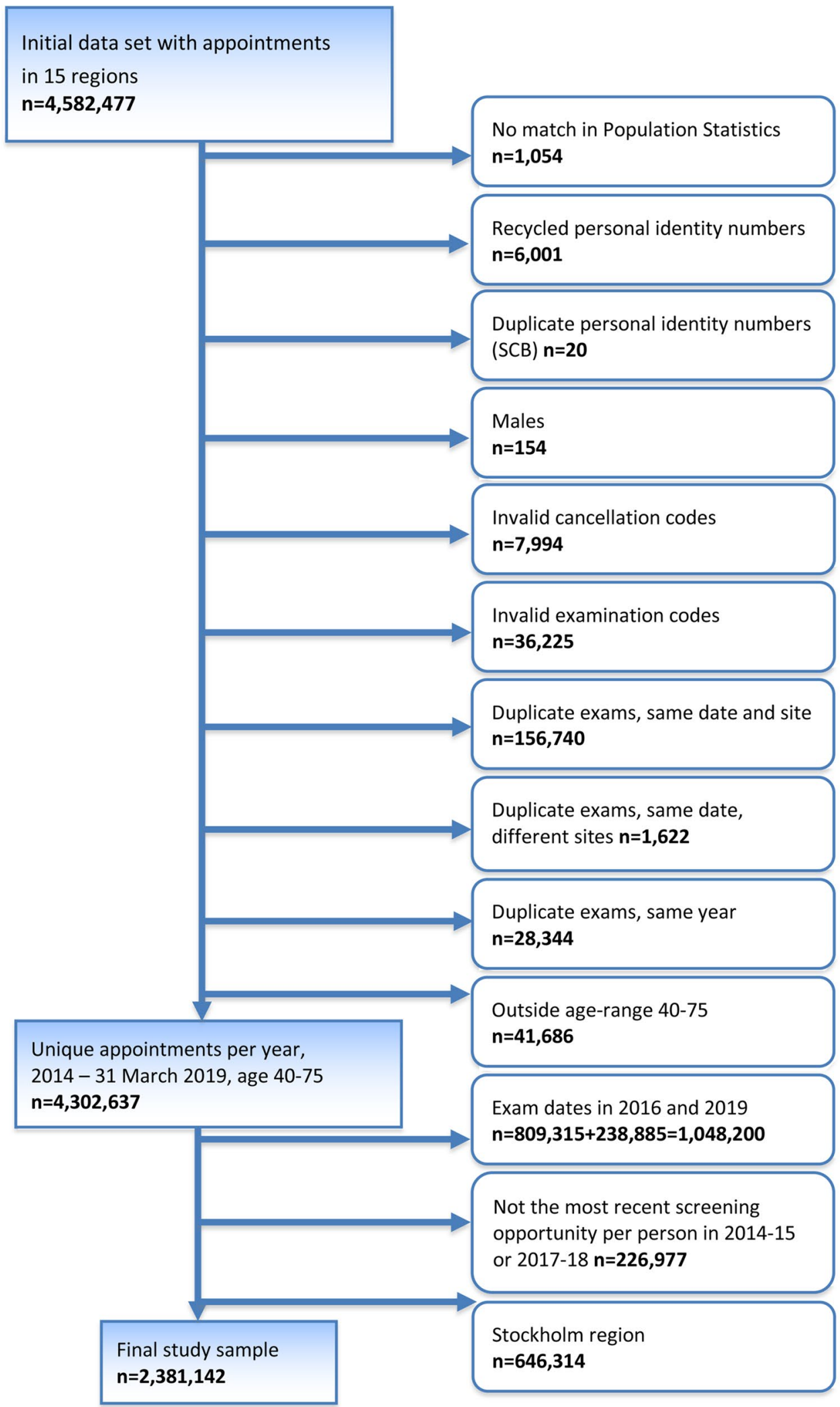

\section{Sociodemographic and program-related variables}

Sociodemographic variables and categorizations are presented in Table 1 and include age group, cohabitation (in which only couples who have children together were categorized as cohabiting), level of education, income (individual 


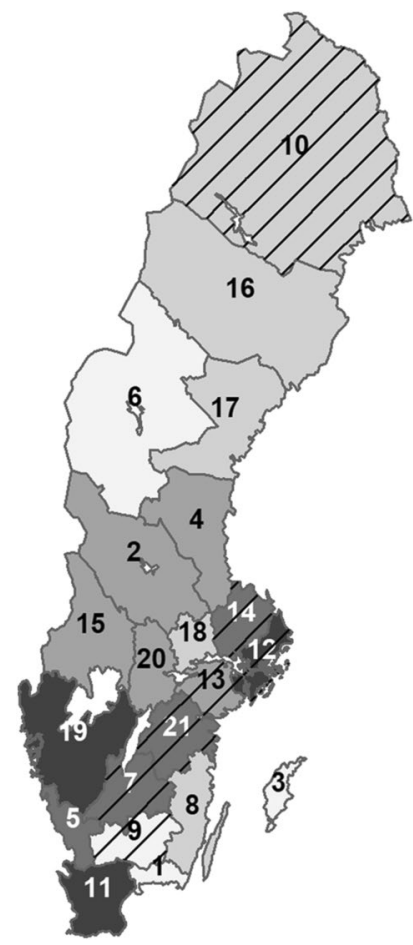

removing the out-of-pocket fee was reported in percentage points with $95 \%$ CIs. We identified six large sociodemographic groups $(n>50,000)$ with attendance below $80 \%$ in 2014-2015 and further examined change in attendance before and after the fee removal in each of these groups and combinations thereof. Since the same women can be included in both time periods, mixed logistic regression was used to account for the correlation of observations within individuals. Results are presented as odds ratios (ORs) and 95\% CIs for mammography attendance in 2017-2018 vs. 2014-2015. We calculated unadjusted estimates, as well as estimates adjusted for the potential confounding effect of various sociodemographic factors. Statistical software used for the analyses were SPSS, version 25, and R, version 4.0.

\section{Results}

The study sample among the 14 included health care regions contained a total of 2,381,142 appointments among 1,350,654 women, with 1,191,609 appointments in 2014-2015 and 1,189,533 appointments in 2017-2018. A total of 1,032,810 women had an appointment in each time period.

Descriptive characteristics of the study sample in each two-year time period are presented in Table 1. In 2014-2015, the mean age at the time of the screening appointment was 56 years; $61 \%$ of women were living with a partner; $16 \%$ had a low level of education; $12 \%$ got their main income from social assistance and benefits and $85 \%$ from employment or retirement income; $74 \%$ owned their home; $84 \%$ were born in Sweden; 25\% lived in a large city or surrounding commuting areas; and $37 \%$ lived in smaller cities and rural areas. Regions with the largest share of the sample were Västra Götaland (28.7\%) and Skåne (22.3\%). Most proportions were stable between time periods; all of the standardized differences were below $10 \%$.

The overall attendance for all regions combined was $83.8 \%$ in $2014-2015$ and $84.1 \%$ in $2017-2018$ (+ 0.3 percentage points, $95 \%$ CI $0.2-0.4$ ). Attendance per region and by sociodemographic factor are presented in Table 2 . There was no change in attendance between 2014-2015 and 2017-2018 in the group of regions with the lowest fees (80-120 SEK) in 2015. A larger increase occurred in the regions that charged 150 SEK than among regions charging 200 SEK. In 2014-2015, Gotland had the lowest attendance (81.0\%), followed by Örebro (82.0\%) and the highest attendance was achieved in Jämtland/Härjedalen (86.1\%), followed by Värmland (85.9\%) and Västmanland (85.5\%). A statistically significant increase in attendance from 2014-2015 to 2017-2018 occurred in seven regions, with the highest increase in Västernorrland (+ 3.1 points), Västerbotten (+ 1.4 points), and Halland (+ 1.3 points). In four regions, there 
Table 1 Sociodemographic and other characteristics of the study sample at the time of the most recent mammography screening appointment in Sweden (2014-2015 and 2017-2018)

\begin{tabular}{|c|c|c|c|c|c|}
\hline \multirow[t]{2}{*}{ Characteristic } & \multicolumn{2}{|c|}{$\begin{array}{l}2014-2015 \\
N=1,191,609\end{array}$} & \multicolumn{2}{|c|}{$\begin{array}{l}2017-2018 \\
N=1,189,533\end{array}$} & \multirow{2}{*}{$\begin{array}{l}\text { Standardized } \\
\text { difference }^{\mathrm{a}} \\
\%\end{array}$} \\
\hline & $N$ & $\%$ & $N$ & $\%$ & \\
\hline Mean age (SD) & 56.12 & $(10.06)$ & 56.37 & $(10.10)$ & 7.87 \\
\hline \multicolumn{6}{|l|}{ Age group (years) } \\
\hline $40-44$ & 192,887 & 16.19 & 185,197 & 15.57 & 1.69 \\
\hline $45-49$ & 186,686 & 15.67 & 176,442 & 14.83 & 2.32 \\
\hline $50-54$ & 172,539 & 14.48 & 188,375 & 15.84 & 3.78 \\
\hline $55-59$ & 164,171 & 13.78 & 162,726 & 13.68 & 0.28 \\
\hline $60-64$ & 163,671 & 13.74 & 159,841 & 13.44 & 0.87 \\
\hline $65-69$ & 173,291 & 14.54 & 159,718 & 13.43 & 3.22 \\
\hline $70-75$ & 138,364 & 11.61 & 157,234 & 13.22 & 4.87 \\
\hline \multicolumn{6}{|l|}{ Cohabitation (living with partner) } \\
\hline Yes & 721,892 & 60.58 & 721,839 & 60.68 & 0.21 \\
\hline No & 464,111 & 38.95 & 464,613 & 39.06 & 0.23 \\
\hline Missing & 5,606 & 0.47 & 3,081 & 0.26 & 3.51 \\
\hline \multicolumn{6}{|l|}{ Level of education } \\
\hline Low (elementary school, $\leq 9$ years) & 193,073 & 16.20 & 171,287 & 14.40 & 5.01 \\
\hline Intermediate (secondary school) & 550,549 & 46.20 & 542,545 & 45.61 & 1.19 \\
\hline High (post-secondary) & 433,252 & 36.36 & 462,339 & 38.87 & 5.18 \\
\hline Missing & 14,735 & 1.24 & 13,362 & 1.12 & 1.05 \\
\hline \multicolumn{6}{|l|}{ Income category $^{\mathrm{b}}$} \\
\hline Lowest (decile 1) & 118,754 & 9.97 & 118,677 & 9.98 & 0.04 \\
\hline Low-medium (decile 2-4) & 355,513 & 29.83 & 355,832 & 29.91 & 0.17 \\
\hline Medium-high (decile 5-10) & 711,736 & 59.73 & 711,944 & 59.85 & 0.25 \\
\hline Missing & 5,606 & 0.47 & 3,080 & 0.26 & 3.51 \\
\hline \multicolumn{6}{|l|}{ Main source of income } \\
\hline Employment & 693,208 & 58.17 & 716,433 & 60.23 & 4.18 \\
\hline Retirement pension & 315,836 & 26.51 & 299,031 & 25.14 & 0.32 \\
\hline Student finance & 4,574 & 0.38 & 4293 & 0.36 & 0.38 \\
\hline Care of a sick child or relative & 5,777 & 0.48 & 6,090 & 0.51 & 0.39 \\
\hline \multicolumn{6}{|l|}{ Social assistance and benefits } \\
\hline Sickness benefit & 25,046 & 2.10 & 25,792 & 2.17 & 0.46 \\
\hline Sickness compensation & 74,548 & 6.26 & 67,550 & 5.68 & 1.19 \\
\hline Unemployment insurance/benefit & 7382 & 0.62 & 6298 & 0.53 & 2.44 \\
\hline Labour market program & 15,125 & 1.27 & 14,261 & 1.20 & 0.64 \\
\hline Financial assistance & 20,339 & 1.71 & 23,107 & 1.94 & 1.76 \\
\hline No income & 24,168 & 2.03 & 23,598 & 1.98 & 0.32 \\
\hline Missing & 5,606 & 0.47 & 3,080 & 0.26 & 3.51 \\
\hline \multicolumn{6}{|l|}{ Home ownership } \\
\hline Yes (house or apartment) & 883,642 & 74.16 & 882,378 & 74.18 & 1.63 \\
\hline No & 280,078 & 23.50 & 287,870 & 24.20 & 0.05 \\
\hline Missing & 27,889 & 2.34 & 19,285 & 1.62 & 5.16 \\
\hline \multicolumn{6}{|l|}{ Country of birth } \\
\hline Sweden & $1,001,904$ & 84.08 & 979,249 & 82.32 & 4.70 \\
\hline Nordic country (except Sweden) & 73,001 & 6.13 & 78,051 & 6.56 & 1.79 \\
\hline Europe (except Sweden and Nordic countries) & 72,832 & 6.11 & 92,728 & 7.80 & 6.62 \\
\hline Other & 43,813 & 3.68 & 39,427 & 3.31 & 1.97 \\
\hline Missing & 59 & 0.00 & 78 & 0.01 & 0.21 \\
\hline \multicolumn{6}{|l|}{ Region } \\
\hline Blekinge & 32,735 & 2.75 & 33,110 & 2.78 & 0.22 \\
\hline
\end{tabular}


Table 1 (continued)

\begin{tabular}{|c|c|c|c|c|c|}
\hline \multirow[t]{2}{*}{ Characteristic } & \multicolumn{2}{|c|}{$\begin{array}{l}2014-2015 \\
N=1,191,609\end{array}$} & \multicolumn{2}{|c|}{$\begin{array}{l}2017-2018 \\
N=1,189,533\end{array}$} & \multirow{2}{*}{$\begin{array}{l}\text { Standardized } \\
\text { difference }^{\mathrm{a}} \\
\%\end{array}$} \\
\hline & $N$ & $\%$ & $N$ & $\%$ & \\
\hline Dalarna & 63,497 & 5.33 & 60,682 & 5.10 & 1.02 \\
\hline Gotland & 13,577 & 1.14 & 13,832 & 1.16 & 0.22 \\
\hline Gävleborg & 62,516 & 5.25 & 60,775 & 5.11 & 0.62 \\
\hline Halland & 64,814 & 5.44 & 65,874 & 5.54 & 0.43 \\
\hline Jämtland/Härjedalen & 25,501 & 2.14 & 26,699 & 2.24 & 0.71 \\
\hline Kalmar & 51,321 & 4.31 & 50,630 & 4.26 & 0.25 \\
\hline Skåne & 265,192 & 22.25 & 271,723 & 22.84 & 1.41 \\
\hline Värmland & 60,219 & 5.05 & 59,993 & 5.04 & 0.05 \\
\hline Västerbotten & 50,257 & 4.22 & 49,076 & 4.13 & 0.46 \\
\hline Västernorrland & 50,328 & 4.22 & 50,988 & 4.29 & 0.31 \\
\hline Västmanland & 53,995 & 4.53 & 47,125 & 3.96 & 2.83 \\
\hline Västra Götaland & 342,100 & 28.71 & 348,371 & 29.29 & 1.27 \\
\hline Örebro & 55,557 & 4.66 & 50,655 & 4.26 & 1.96 \\
\hline \multicolumn{6}{|l|}{ Type of municipality } \\
\hline Large cities $(>200,000)^{\mathrm{c}}$ & 294,396 & 24.71 & 305,857 & 25.71 & 2.32 \\
\hline Mid-sized cities $(50,000-200,000)^{\mathrm{d}}$ & 455,161 & 38.20 & 451,125 & 37.92 & 0.56 \\
\hline Smaller cities, towns, and rural areas & 436,446 & 36.63 & 429,897 & 36.14 & 1.01 \\
\hline Missing & 5,606 & 0.47 & 2,654 & 0.22 & 4.21 \\
\hline \multicolumn{6}{|l|}{ Out-of-pocket fee (2015) in SEK ${ }^{\mathrm{e}}$} \\
\hline 80 & 55,557 & 4.66 & 50,655 & 4.26 & 1.96 \\
\hline 100 & 393,421 & 33.02 & 399,001 & 33.54 & 1.12 \\
\hline 120 & 297,927 & 25.00 & 304,833 & 25.63 & 1.44 \\
\hline 150 & 204,140 & 17.13 & 204,243 & 17.17 & 0.10 \\
\hline 200 & 240,564 & 20.19 & 230,801 & 19.40 & 1.97 \\
\hline \multicolumn{6}{|l|}{ Year of scheduled appointment } \\
\hline 2014 & 542,148 & 45.50 & & & \\
\hline 2015 & 649,461 & 54.50 & & & \\
\hline 2017 & & & 542,602 & 45.61 & \\
\hline 2018 & & & 646,931 & 54.39 & \\
\hline
\end{tabular}

${ }^{\text {a }}$ The difference between the groups divided by the pooled standard deviation; a value greater than $10 \%$ is interpreted as a meaningful difference

${ }^{\mathrm{b}}$ Income categories for 2014-15: Lowest: $\leq$ 92,700 SEK; low-medium: 92,800-150,400 SEK; mediumhigh: $\geq 150,500$ SEK. Income categories for 2017-18: Lowest: $\leq$ 98,300 SEK; low-medium: 98,400162,700 SEK; medium-high: $\geq 162,800$ SEK

${ }^{\mathrm{c}}$ Includes commuting zone

${ }^{\mathrm{d}}$ Includes neighboring municipalities

e 80 (Örebro), 100 (Kalmar and Västra Götaland), 120 (Blekinge and Skåne), 150 (Dalarna, Halland, Jämtland, and Västernorrland), 200 (Gotland, Gävleborg, Värmland, Västerbotten, and Västmanland)

was a statistically significant decrease in attendance, with the largest decrease in Örebro ( -1.3 points) and Blekinge ( -1.1 points). Attendance according to age groups varied from $82.2 \%$ among women in their forties to $86.0 \%$ among women in their sixties in 2014-2015. The largest increase over time was found among women 70-75 years of age (+ 1.0 points). In 2014-2015, attendance was least prevalent among women who lived without a partner (76.9\%), who had a low level of education level (78.0\%), who were in the lowest income decile (71.8\%), whose main source of income was not employment or retirement pension (e.g., 68.8\% among women with social assistance or benefits and 56.0\% among those with no income), who did not own their home (73.5\%), and who were not born in Sweden and especially among those born outside of the Nordic countries (70.8\%).

When assessing how the time period associated with attendance among all women in the study sample, the odds of attending were not statistically higher in 2017-2018 than in 2014-2015, neither in an unadjusted analysis (OR 1.01, 95\% CI 0.99-1.02) nor when adjusting for region, age, 
Table 2 Mammography screening attendance with 95\% confidence intervals (CI) and change in percentage points (PP) from 2014-2015 to $2017-2018$ by sociodemographic factor $(\mathrm{n}=$ number of attenders)

\begin{tabular}{|c|c|c|c|c|c|c|c|c|c|c|c|}
\hline \multirow[t]{2}{*}{ Variable } & \multicolumn{4}{|c|}{$\begin{array}{l}2014-2015 \\
(N=1,191,609)\end{array}$} & \multicolumn{4}{|c|}{$\begin{array}{l}2017-2018 \\
(N=1,189,533)\end{array}$} & \multicolumn{3}{|c|}{ Change $(95 \% \mathrm{CI})$} \\
\hline & $n$ & $\%$ & Lower CI & Upper CI & $n$ & $\%$ & Lower CI & Upper CI & $\mathrm{PP}$ & Lower CI & Upper CI \\
\hline Total & 998,533 & 83.80 & 83.73 & 83.86 & 999,913 & 84.06 & 83.99 & 84.13 & 0.26 & 0.17 & 0.36 \\
\hline \multicolumn{12}{|l|}{ Age group } \\
\hline $40-44$ & 158,851 & 82.35 & 82.18 & 82.52 & 153,410 & 82.84 & 82.66 & 83.01 & 0.48 & 0.24 & 0.72 \\
\hline $45-49$ & 153,046 & 81.98 & 81.81 & 82.15 & 145,817 & 82.64 & 82.47 & 82.82 & 0.66 & 0.41 & 0.91 \\
\hline $50-54$ & 142,228 & 82.43 & 82.25 & 82.61 & 155,948 & 82.79 & 82.62 & 82.96 & 0.35 & 0.11 & 0.60 \\
\hline $55-59$ & 138,116 & 84.13 & 83.95 & 84.31 & 136,605 & 83.95 & 83.77 & 84.13 & -0.18 & -0.43 & 0.07 \\
\hline $60-64$ & 139,594 & 85.29 & 85.12 & 85.46 & 136,189 & 85.20 & 85.03 & 85.38 & -0.09 & -0.33 & 0.16 \\
\hline $65-69$ & 150,219 & 86.69 & 86.53 & 86.85 & 138,083 & 86.45 & 86.29 & 86.62 & -0.23 & -0.46 & 0.00 \\
\hline $70-75$ & 116,479 & 84.18 & 83.99 & 84.38 & 133,861 & 85.13 & 84.96 & 85.31 & 0.95 & 0.69 & 1.21 \\
\hline \multicolumn{12}{|l|}{ Cohabitation } \\
\hline Yes & 639,756 & 88.62 & 88.55 & 88.70 & 639,126 & 88.54 & 88.47 & 88.61 & -0.08 & -0.18 & 0.02 \\
\hline No & 357,050 & 76.93 & 76.81 & 77.05 & 359,585 & 77.39 & 77.27 & 77.51 & 0.46 & 0.29 & 0.63 \\
\hline \multicolumn{12}{|l|}{ Level of education } \\
\hline Low & 150,608 & 78.01 & 77.82 & 78.19 & 132,025 & 77.08 & 76.88 & 77.28 & -0.93 & -1.20 & -0.66 \\
\hline Intermediate & 465,077 & 84.48 & 84.38 & 84.57 & 458,064 & 84.43 & 84.33 & 84.53 & -0.05 & -0.18 & 0.09 \\
\hline High & 376,740 & 86.96 & 86.86 & 87.06 & 403,215 & 87.21 & 87.12 & 87.31 & 0.26 & 0.12 & 0.39 \\
\hline \multicolumn{12}{|l|}{ Income } \\
\hline Lowest decile & 85,270 & 71.80 & 71.55 & 72.06 & 85,670 & 72.19 & 71.93 & 72.44 & 0.38 & 0.02 & 0.74 \\
\hline Decile 2-4 & 289,236 & 81.36 & 81.23 & 81.49 & 290,381 & 81.61 & 81.48 & 81.73 & 0.25 & 0.07 & 0.43 \\
\hline Decile $5-10$ & 622,300 & 87.43 & 87.36 & 87.51 & 622,661 & 87.46 & 87.38 & 87.54 & 0.03 & -0.08 & 0.13 \\
\hline \multicolumn{12}{|l|}{ Main source of income } \\
\hline Employment/retirement & 877,908 & 87.00 & 86.94 & 87.07 & 882,134 & 86.87 & 86.80 & 86.94 & -0.13 & -0.23 & -0.04 \\
\hline Student finance & 3,463 & 75.71 & 74.47 & 76.95 & 3,290 & 76.64 & 75.37 & 77.90 & 0.93 & -0.85 & 2.70 \\
\hline Care of sick child/relative & 3,952 & 68.41 & 67.21 & 69.61 & 4,415 & 72.50 & 71.37 & 73.62 & 4.09 & 2.45 & 5.73 \\
\hline Social assistance/benefits & 97,949 & 68.77 & 68.52 & 69.01 & 95,447 & 69.67 & 69.42 & 69.91 & 0.90 & 0.56 & 1.24 \\
\hline No income & 13,534 & 56.00 & 55.37 & 56.63 & 13,426 & 56.89 & 56.26 & 57.53 & 0.89 & 0.01 & 1.78 \\
\hline \multicolumn{12}{|l|}{ Home ownership } \\
\hline Yes & 774,600 & 87.66 & 87.59 & 87.73 & 773,668 & 87.68 & 87.61 & 87.75 & 0.02 & -0.08 & 0.12 \\
\hline No & 205,775 & 73.47 & 73.31 & 73.63 & 213,390 & 74.13 & 73.97 & 74.29 & 0.66 & 0.43 & 0.89 \\
\hline \multicolumn{12}{|l|}{ Country of birth } \\
\hline Sweden & 861,164 & 85.95 & 85.88 & 86.02 & 845,066 & 86.30 & 86.23 & 86.37 & 0.34 & 0.25 & 0.44 \\
\hline Nordic country & 34,017 & 77.64 & 77.25 & 78.03 & 30,975 & 78.56 & 78.16 & 78.97 & 0.92 & 0.36 & 1.48 \\
\hline Europe & 51,696 & 70.82 & 70.49 & 71.15 & 55,945 & 71.68 & 71.36 & 71.99 & 0.86 & 0.41 & 1.32 \\
\hline Other & 51,625 & 70.88 & 70.55 & 71.21 & 67,883 & 73.21 & 72.92 & 73.49 & 2.32 & 1.89 & 2.76 \\
\hline \multicolumn{12}{|l|}{ Region } \\
\hline Blekinge & 28,741 & 87.80 & 87.44 & 88.15 & 28,715 & 86.73 & 86.36 & 87.09 & -1.07 & -1.58 & -0.56 \\
\hline Dalarna & 53,141 & 83.69 & 83.40 & 83.98 & 51,165 & 84.32 & 84.03 & 84.61 & 0.63 & 0.22 & 1.03 \\
\hline Gotland & 11,002 & 81.03 & 80.37 & 81.69 & 11,368 & 82.19 & 81.55 & 82.82 & 1.15 & 0.23 & 2.07 \\
\hline Gävleborg & 52,158 & 83.43 & 83.14 & 83.72 & 50,837 & 83.65 & 83.35 & 83.94 & 0.22 & -0.20 & 0.63 \\
\hline Halland & 55,339 & 85.38 & 85.11 & 85.65 & 57,119 & 86.71 & 86.45 & 86.97 & 1.33 & 0.95 & 1.70 \\
\hline Jämtland/Härjedalen & 21,969 & 86.15 & 85.73 & 86.57 & 22,963 & 86.01 & 85.59 & 86.42 & -0.14 & -0.74 & 0.45 \\
\hline Kalmar & 43,380 & 84.53 & 84.21 & 84.84 & 42,708 & 84.35 & 84.04 & 84.67 & -0.17 & -0.62 & 0.27 \\
\hline Skåne & 218,281 & 82.31 & 82.17 & 82.46 & 222,339 & 81.83 & 81.68 & 81.97 & -0.48 & -0.69 & -0.28 \\
\hline Värmland & 51,733 & 85.91 & 85.63 & 86.19 & 51,355 & 85.60 & 85.32 & 85.88 & -0.31 & -0.70 & 0.09 \\
\hline Västerbotten & 42,857 & 85.28 & 84.97 & 85.59 & 42,554 & 86.71 & 86.41 & 87.01 & 1.43 & 1.00 & 1.87 \\
\hline Västernorrland & 42,277 & 84.00 & 83.68 & 84.32 & 44,425 & 87.13 & 86.84 & 87.42 & 3.13 & 2.69 & 3.56 \\
\hline Västmanland & 46,155 & 85.48 & 85.18 & 85.78 & 40,509 & 85.96 & 85.65 & 86.27 & 0.48 & 0.05 & 0.91 \\
\hline
\end{tabular}


Table 2 (continued)

\begin{tabular}{|c|c|c|c|c|c|c|c|c|c|c|c|}
\hline \multirow[t]{2}{*}{ Variable } & \multicolumn{4}{|c|}{$\begin{array}{l}2014-2015 \\
(N=1,191,609)\end{array}$} & \multicolumn{4}{|c|}{$\begin{array}{l}2017-2018 \\
(N=1,189,533)\end{array}$} & \multicolumn{3}{|c|}{ Change $(95 \% \mathrm{CI})$} \\
\hline & $n$ & $\%$ & Lower CI & Upper CI & $n$ & $\%$ & Lower CI & Upper CI & PP & Lower CI & Upper CI \\
\hline Västra Götaland & 285,926 & 83.58 & 83.46 & 83.70 & 292,982 & 84.10 & 83.98 & 84.22 & 0.52 & 0.35 & 0.69 \\
\hline Örebro & 45,574 & 82.03 & 81.71 & 82.35 & 40,874 & 80.69 & 80.35 & 81.03 & -1.34 & -1.81 & -0.87 \\
\hline \multicolumn{12}{|l|}{ Type of municipality } \\
\hline Large cities & 240,174 & 81.58 & 81.44 & 81.72 & 250,738 & 81.98 & 81.84 & 82.12 & 0.40 & 0.20 & 0.59 \\
\hline Mid-sized cities & 384,838 & 84.55 & 84.44 & 84.65 & 381,547 & 84.58 & 84.47 & 84.68 & 0.03 & -0.12 & 0.18 \\
\hline Smaller cities/rural areas & 371,794 & 85.19 & 85.08 & 85.29 & 366,731 & 85.31 & 85.20 & 85.41 & 0.12 & -0.03 & 0.27 \\
\hline \multicolumn{12}{|l|}{ Out-of-pocket fee (2015) } \\
\hline 80 & 45,574 & 82.03 & 81.71 & 82.35 & 40,874 & 80.69 & 80.35 & 81.03 & -1.34 & -1.81 & -0.87 \\
\hline 100 & 329,306 & 83.70 & 83.59 & 83.82 & 335,690 & 84.13 & 84.02 & 84.25 & 0.43 & 0.27 & 0.59 \\
\hline 120 & 247,022 & 82.91 & 82.78 & 83.05 & 251,054 & 82.36 & 82.22 & 82.49 & -0.56 & -0.75 & -0.36 \\
\hline 150 & 172,726 & 84.61 & 84.46 & 84.77 & 175,672 & 86.01 & 85.86 & 86.16 & 1.40 & 1.18 & 1.62 \\
\hline 200 & 203,905 & 84.76 & 84.62 & 84.90 & 196,623 & 85.19 & 85.05 & 85.34 & 0.43 & 0.23 & 0.63 \\
\hline
\end{tabular}

cohabitation, education, income, main source of income, home ownership, and country of birth (OR 1.00, 95\% CI 0.99-1.01).

Table 3 presents attendance before and after the fee removal in sociodemographic sub-groups at risk of low attendance. Although the change between time periods was statistically significant in all six of the main sub-groups, it was small and only exceeded one percentage point for those born in non-Nordic countries-where attendance increased by 1.7 points from 2014-2015 to 2017-2018. This effect remained statistically significant in the multivariable analysis. Among women with a low level of education, attendance decreased by 0.9 points and this effect persisted in the multivariable analysis. Attendance increased from 59.2 to $62.0 \%$ (+ 2.8 points, $95 \%$ CI 2.2-3.4) among women with a combination of any four or more risk factors for low attendance, but did not change substantially among those with three or fewer risk factors. When risk factors were combined, the groups decreased considerably in size and women with four or more risk factors constituted only $3.2 \%$ of all women invited during 2014-2015. When two specific risk factors were combined, the lowest attendance in 2014-2015 was found among women who had the lowest income and were living without a partner $(53.0 \%)$ and among women whose main source of income was social assistance and benefits and either lived without a partner $(60.9 \%)$ or did not own their home $(61.2 \%)$. The biggest increase in attendance was found among non-Nordic women with the lowest income (+ 2.9 points, 95\% CI 2.3-3.6). Other larger sub-groups where attendance increased by more than two points were women born outside of the Nordic countries who were either living alone (+2.1 points, 95\% CI 1.6-2.6), did not own their home (+2.0 points, $95 \%$ CI 1.5-2.5), or whose main source of income was social assistance and benefits $(+2.1$ points,
95\% CI 1.5-2.7) and women whose main source of income was social assistance and benefits and who did not own their home (+ 2.3 points, 95\% CI 1.8-2.8). In all combined risk groups, the association between time period and attendance remained statistically significant in the multivariable analysis.

\section{Discussion}

In this longitudinal population-based register study of mammography screening attendance in Sweden, we found that overall attendance remained relatively stable at $84 \%$ after removal of the out-of-pocket fee in 2016, but rose by 2.9 percentage points among non-Nordic women with the lowest income (from 62.9 to $65.8 \%$ ) and by 2.8 points among women with four or more risk factors for low attendance (from 59.2 to $62.0 \%$ ).

To our knowledge, no other European studies have reported on the effect of removing the fee for mammography screening on screening attendance, except for a study in Stockholm showing an increase of two percentage points after removing the out-of-pocket fee in 2012 [15]. However, a study in Finland found that the likelihood of screening attendance decreased after a fee was introduced, independently of socioeconomic status [20]. A Swedish randomized study examining the effect of offering free cervical cancer screening exams to women in socioeconomically disadvantaged areas failed to establish a statistically significant effect on attendance [21].

Although some US research has demonstrated a positive effect of reducing or removing the out-of-pocket fee on screening attendance [12-14], more recent studies investigating the effect of cost-share removal among Medicare 
Table 3 Effect of time period on mammography screening attendance in selected sub-groups with low attendance. Mixed logistic regression analysis of attendance in 2017-2018 vs. 2014-2015

\begin{tabular}{|c|c|c|c|c|c|c|c|c|c|}
\hline \multirow[t]{2}{*}{ Sub-group } & \multicolumn{2}{|c|}{ 2014-2015 } & \multicolumn{2}{|c|}{ 2017-2018 } & \multicolumn{3}{|c|}{ Change (95\% CI) } & \multirow{2}{*}{$\begin{array}{l}\text { Unadjusted } \\
\text { OR (95\% CI) }\end{array}$} & \multirow{2}{*}{$\begin{array}{l}\text { Adjusted } \\
\text { OR }(95 \% \text { CI })\end{array}$} \\
\hline & $n$ & $\%$ & $n$ & $\%$ & PP & Lower CI & Upper CI & & \\
\hline \multicolumn{10}{|l|}{ Risk factor for low attendance } \\
\hline Living without partner (alone) & 357,050 & 76.93 & 359,585 & 77.39 & 0.46 & 0.29 & 0.63 & $1.02(1.01-1.03)$ & $1.01(0.99-1.02)^{\mathrm{a}}$ \\
\hline Low level of education & 150,608 & 78.01 & 132,025 & 77.08 & -0.93 & -1.20 & -0.66 & $0.94(0.92-0.96)$ & $0.95(0.93-0.97)^{\mathrm{b}}$ \\
\hline Lowest income & 85,270 & 71.80 & 85,670 & 72.19 & 0.38 & 0.02 & 0.74 & $1.02(0.99-1.04)$ & $1.02(1.00-1.05)^{\mathrm{c}}$ \\
\hline Social assistance/benefits & 97,949 & 68.77 & 95,447 & 69.67 & 0.90 & 0.56 & 1.24 & $1.05(1.03-1.07)$ & $1.04(1.02-1.06)^{\mathrm{d}}$ \\
\hline Not owning home & 205,775 & 73.47 & 213,390 & 74.13 & 0.66 & 0.43 & 0.89 & $1.04(1.02-1.05)$ & $1.01(1.00-1.03)^{\mathrm{e}}$ \\
\hline Non-Nordic & 103,321 & 70.85 & 123,828 & 72.51 & 1.66 & 1.34 & 1.97 & $1.08(1.06-1.10)$ & $1.05(1.03-1.07)^{\mathrm{f}}$ \\
\hline \multicolumn{10}{|l|}{ Index of the above 6 risk factors ${ }^{g}$} \\
\hline 0 risk factor & 411,288 & 91.63 & 413,250 & 92.03 & 0.41 & 0.29 & 0.52 & $1.12(1.08-1.15)$ & $1.11(1.07-1.15)$ \\
\hline 1 & 313,432 & 86.11 & 311,851 & 86.11 & 0.00 & -0.16 & 0.16 & $0.98(0.95-1.01)$ & $0.97(0.93-1.00)$ \\
\hline 2 & 176,436 & 78.15 & 173,189 & 77.95 & -0.20 & -0.44 & 0.05 & $0.98(0.97-1.00)$ & $0.98(0.96-1.00)$ \\
\hline 3 & 65,709 & 66.04 & 66,390 & 66.83 & 0.79 & 0.37 & 1.20 & $1.03(1.01-1.06)$ & $1.03(1.01-1.06)$ \\
\hline 4 & 22,675 & 58.61 & 24,727 & 61.32 & 2.71 & 2.03 & 3.39 & $1.12(1.08-1.16)$ & $1.13(1.10-1.18)$ \\
\hline 5 & 8,116 & 60.89 & 9,398 & 63.71 & 2.82 & 1.69 & 3.96 & $1.19(1.10-1.28)$ & $1.23(1.14-1.33)$ \\
\hline 6 & 877 & 59.14 & 1,108 & 62.32 & 3.18 & -0.19 & 6.55 & $1.20(0.97-1.49)$ & $1.22(0.98-1.52)$ \\
\hline 4 or more & 31,668 & 59.19 & 35,233 & 61.97 & 2.78 & 2.20 & 3.36 & $1.12(1.09-1.16)$ & $1.14(1.10-1.17)$ \\
\hline 5 or 6 & 8,993 & 60.71 & 10,506 & 63.56 & 2.85 & 1.77 & 3.92 & $1.13(1.05-1.21)$ & $1.21(1.13-1.30)$ \\
\hline \multicolumn{10}{|l|}{ Selected combinations ${ }^{\mathrm{h}}$} \\
\hline Lowest income + living alone & 15,854 & 53.02 & 16,989 & 54.81 & 1.79 & 1.00 & 2.58 & $1.07(1.03-1.11)$ & $1.07(1.02-1.12)^{\mathrm{i}}$ \\
\hline Lowest income + social assist & 18,462 & 64.83 & 20,734 & 67.33 & 2.50 & 1.73 & 3.26 & $1.17(1.12-1.22)$ & $1.15(1.09-1.21)^{\mathrm{j}}$ \\
\hline Lowest income + not owning & 24,956 & 64.28 & 27,945 & 66.26 & 1.98 & 1.32 & 2.63 & $1.09(1.06-1.13)$ & $1.10(1.05-1.14)^{\mathrm{k}}$ \\
\hline Non-Nordic + lowest income & 26,128 & 62.87 & 31,038 & 65.79 & 2.92 & 2.29 & 3.55 & $1.14(1.10-1.17)$ & $1.17(1.12-1.21)^{1}$ \\
\hline Non-Nordic + living alone & 39,079 & 64.13 & 47,236 & 66.21 & 2.09 & 1.57 & 2.60 & $1.09(1.06-1.12)$ & $1.06(1.02-1.09)^{\mathrm{m}}$ \\
\hline Non-Nordic + social assist & 26,803 & 66.85 & 29,459 & 68.96 & 2.11 & 1.47 & 2.75 & $1.10(1.06-1.14)$ & $1.13(1.08-1.17)^{\mathrm{n}}$ \\
\hline Non-Nordic + not owning & 44,735 & 66.56 & 54,933 & 68.58 & 2.02 & 1.54 & 2.50 & $1.10(1.07-1.13)$ & $1.07(1.04-1.10)^{\mathrm{o}}$ \\
\hline Social assist + living alone & 48,165 & 60.86 & 48,068 & 62.60 & 1.74 & 1.26 & 2.22 & $1.08(1.05-1.11)$ & $1.07(1.04-1.10)^{\mathrm{p}}$ \\
\hline Social assist + not owning & 41,403 & 61.19 & 42,936 & 63.51 & 2.32 & 1.80 & 2.83 & $1.11(1.08-1.14)$ & $1.08(1.05-1.11)^{\mathrm{q}}$ \\
\hline
\end{tabular}

ORs Odds Ratios, CIs 95\% confidence intervals, $n$ number of attenders, $P P$ percentage points

${ }^{a}$ Estimates were adjusted for region, age group, education, income, main source of income, home ownership, and country of birth

${ }^{b}$ Estimates were adjusted for region, age group, cohabitation, income, main source of income, home ownership, and country of birth

${ }^{c}$ Estimates were adjusted for region, age group, cohabitation, education, main source of income, home ownership, and country of birth

${ }^{\mathrm{d}}$ Estimates were adjusted for region, age group, cohabitation, education, income, home ownership, and country of birth

${ }^{\mathrm{e}}$ Estimates were adjusted for region, age group, cohabitation, education, income, main source of income, and country of birth

${ }^{\mathrm{f}}$ Estimates were adjusted for region, age group, cohabitation, education, income, main source of income, and home ownership

${ }^{\mathrm{g}}$ Estimates were adjusted for region and age group

${ }^{\mathrm{h}} \mathrm{A}$ combination of two risk factors was selected for inclusion where the change exceeded one percentage point and the change in the combined group exceeded the change observed for either individual risk factor. Estimates were adjusted for region, age group, and risk variables not selected for respective subgroup

${ }^{\mathrm{i}}$ Estimates were adjusted for region, age group, education, main source of income, home ownership, and country of birth

${ }^{\mathrm{j}}$ Estimates were adjusted for region, age group, cohabitation, education, home ownership, and country of birth

${ }^{\mathrm{k}}$ Estimates were adjusted for region, age group, cohabitation, education, main source of income, and country of birth

${ }^{1}$ Estimates were adjusted for region, age group, cohabitation, education, main source of income, and home ownership

${ }^{\mathrm{m}}$ Estimates were adjusted for region, age group, education, income, main source of income, and home ownership

${ }^{\mathrm{n}}$ Estimates were adjusted for region, age group, cohabitation, education, income, and home ownership

${ }^{\circ}$ Estimates were adjusted for region, age group, cohabitation, education, income, and main source of income

${ }^{\mathrm{p}}$ Estimates were adjusted for region, age group, education, income, home ownership, and country of birth

${ }^{\mathrm{q}}$ Estimates were adjusted for region, age group, cohabitation, education, income, and country of birth 
beneficiaries show conflicting results. Some studies found no improvement in uptake in general [22-24], nor in low socioeconomic groups [23, 24], whereas others found an increase in general attendance $[25,26]$, as well as among unmarried women [25], but not in areas with lower education [26]. Yet another study detected a smaller decrease in attendance in the intervention group compared to a control group, but found no difference by neighborhood socioeconomic status [27].

The lack of change in attendance in the present study is somewhat surprising, considering that the odds of viewing the screening exam as too expensive were threefold among non-attenders compared to attenders according to a previous Swedish cross-sectional study [28]. However, such an opinion, no matter how strong, is just one of many factors and may not ultimately be the deciding one. Other rationales and life circumstances play into the complex decision-making process that some qualitative research studies have depicted $[29,30]$. The actual out-of-pocket fee was fairly modest, and there could be other inconveniences and costs that influence the decision to attend and remain obstacles to attendance, especially among socioeconomically vulnerable groups. Such obstacles might include transportation to the screening site, making the effort to reschedule, and taking time off from work [31]. This may explain why only removing the out-of-pocket cost does not appear sufficient to impact uptake in these groups.

\section{Strengths and limitations}

Removal of the screening fee was implemented simultaneously in all health care regions included in this study. However, the absence of control groups or a staggered design, and the lack of means to adequately control for other changes and interventions that may have been introduced in different regions during the study period, limit our ability to evaluate a causal effect of introducing free mammography screening on screening attendance. Another requirement for establishing a causal relationship is evidence of a dose-response relationship, which our results do not demonstrate. Although no change in average attendance was noticeable among regions charging 80-120 SEK, a larger average increase occurred among regions that charged 150 SEK than among those charging 200. An absence of a correlation between the fee and the attendance is corroborated by a Swedish study examining mammography screening attendance in 1995-1996 [4].

This study assesses screening attendance up to and including 2018, i.e., two years after eliminating the screening fee, which may not have been a sufficient amount of time for change to occur. There was no standardized process of informing women about the fee removal across regionsalthough a survey among the regions indicated that most of them at least included information about the screening exam being free of charge in the letter of invitation-and the level of awareness of this change in the study population is unknown. Such awareness is a necessary condition for establishing a causal effect on attendance.

The screening attendance, based on the actual appointment date within each time period 2014-2015 and 2017-2018, that is presented in this study, might be somewhat higher than screening attendance based on the primary appointment date specified in the invitation. In about $45 \%$ of the cases, these dates were the same (based on data for 2017 and 2018), but a majority of the primary appointment dates were rescheduled. For 2017 and 2018, we had relatively complete data for the primary appointment date, but not for 2014 and 2015. For comparative reasons, we had to use the same definition of attendance for both time periods. Among those who were invited to be screened during 2017-2018, $82.8 \%$ attended within 90 days of the primary appointment, which is 1.3 percentage points lower than the attendance rate that we present. However, we believe that this difference would have been similar in size for the time period 2014-2015.

Despite these limitations, our population study has many strengths, including its large size, covering the majority of Sweden's regional mammography programs. While many studies may struggle with low response rates and selection bias and rely on self-reported data for both exposure and outcome measures, we obtained this information from high-quality register data, originating from several different official sources with high coverage [16], thus minimizing measurement error and misclassification.

\section{Conclusion}

Screening attendance did not undergo any important increase after implementing free screening, although attendance among some sociodemographic groups increased by almost three percentage points after the policy change. These findings may apply to similar population-based screening programs in countries with universal health care, high screening uptake, and small out-of-pocket fees.

Acknowledgements The authors are grateful to Gabriel Cetin, Erik Fornander, and Anna Löfborg at Sectra Sverige AB for their work extracting our data, to Fredrik Sandin at the Regional Cancer Center Uppsala Örebro, and to all the regional mammography programs for assistance in the data collection process. This study was commissioned and funded by the Swedish Agency for Health and Care Services Analysis.

Funding Open access funding provided by Lund University.

Data availability The data can be made available upon reasonable request. 


\section{Declarations}

Conflict of interest The authors declare that they have no conflict of interest.

Open Access This article is licensed under a Creative Commons Attribution 4.0 International License, which permits use, sharing, adaptation, distribution and reproduction in any medium or format, as long as you give appropriate credit to the original author(s) and the source, provide a link to the Creative Commons licence, and indicate if changes were made. The images or other third party material in this article are included in the article's Creative Commons licence, unless indicated otherwise in a credit line to the material. If material is not included in the article's Creative Commons licence and your intended use is not permitted by statutory regulation or exceeds the permitted use, you will need to obtain permission directly from the copyright holder. To view a copy of this licence, visit http://creativecommons.org/licenses/by/4.0/.

\section{References}

1. Deandrea S, Molina-Barceló A, Uluturk A, Moreno J, Neamtiu L, Peiró-Pérez R, Saz-Parkinson Z, Lopez-Alcalde J, Lerda D, Salas D (2016) Presence, characteristics and equity of access to breast cancer screening programmes in 27 European countries in 2010 and 2014. Results from an international survey. Prev Med 91:250-263

2. Perry N, Broeders M, de Wolf C, Törnberg S, Holland R, von Karsa L (2008) European guidelines for quality assurance in breast cancer screening and diagnosis. Fourth edition-summary document. Ann Oncol 19:614-622

3. National Board of Health and Welfare (2014) Screening för bröstcancer - Rekommendation och bedömningsunderlag [Breast cancer screening - Recommendation and basis for decision]. National Board of Health and Welfare.

4. Olsson S, Andersson I, Karlberg I, Bjurstam N, Frodis E, Håkansson $S$ (2000) Implementation of service screening with mammography in Sweden: from pilot study to nationwide programme. J Med Screen 7:14-18

5. Lagerlund M, Maxwell AE, Bastani R, Thurfjell E, Ekbom A, Lambe M (2002) Sociodemographic predictors of non-attendance at invitational mammography screening - a population-based register study (Sweden). Cancer Causes Control 13:73-82

6. Lagerlund M, Merlo J, Vicente RP, Zackrisson S (2015) Does the neighborhood area of residence influence non-attendance in an urban mammography screening program? A multilevel study in a Swedish city. PLoS ONE 10:e0140244s

7. Zackrisson S, Andersson I, Manjer J, Janzon L (2004) Non-attendance in breast cancer screening is associated with unfavourable socio-economic circumstances and advanced carcinoma. Int $\mathrm{J}$ Cancer 108:754-760

8. Zackrisson S, Lindström M, Moghaddassi M, Andersson I, Janzon L (2007) Social predictors of non-attendance in an urban mammographic screening programme: a multilevel analysis. Scand J Public Health 35:548-554

9. Lagerlund M, Sontrop J, Zackrisson S (2014) Psychosocial factors and attendance at a population-based mammography screening program in a cohort of Swedish women. BMC Womens Health $14: 33$

10. Lagerlund M, Sparén P, Thurfjell E, Ekbom A, Lambe M (2000) Predictors of non-attendance in a population-based mammography screening programme; socio-demographic factors and aspects of health behaviour. Eur J Cancer Prev 9:25-33
11. National Board of Health and Welfare (2016) HSLF-FS 2016:63. Socialstyrelsens föreskrifter och allmänna råd om avgiftsfri screening för bröstcancer med mammografi. [National regulations and guidelines regarding free breast cancer screening with mammography]. In: Welfare NBoHa, ed. Stockholm, Sweden

12. Masi CM, Blackman DJ, Peek ME (2007) Interventions to enhance breast cancer screening, diagnosis, and treatment among racial and ethnic minority women. Med Care Res Rev 64:195S-242S

13. Spadea T, Bellini S, Kunst A, Stirbu I, Costa G (2010) The impact of interventions to improve attendance in female cancer screening among lower socioeconomic groups: a review. Prev Med 50:159-164

14. Stone EG, Morton SC, Hulscher ME, Maglione MA, Roth EA, Grimshaw JM, Mittman BS, Rubenstein LV, Rubenstein LZ, Shekelle PG (2002) Interventions that increase use of adult immunization and cancer screening services: a meta-analysis. Ann Intern Med 136:641-651

15. Törnberg S, Lidbrink E, Henriksson R (2014) Avgiftsfri mammografi får fler att komma till undersökning. Studie i Stockholms län visar på bra effekt i socioekonomiskt svaga områden [Free of charge mammography gets more people to the examination. Study in Stockholm County shows good efficacy in socioeconomically disadvantaged areas]. Lakartidningen 111:278-281

16. Ludvigsson JF, Svedberg P, Olén O, Bruze G, Neovius M (2019) The longitudinal integrated database for health insurance and labour market studies (LISA) and its use in medical research. Eur J Epidemiol 34:423-437

17. Ludvigsson JF, Almqvist C, Bonamy AK, Ljung R, Michaëlsson K, Neovius M, Stephansson O, Ye W (2016) Registers of the Swedish total population and their use in medical research. Eur J Epidemiol 31:125-136

18. Palmelius S (2019) Det statistiska registrets framställning och kvalietet - geografidatabasen [Rendering and quality of the statistical register - Geodatabase]. SCB. https://www.scb.se/conte ntassets/222e45b854a242aa899111ce753d1a52/ov0100_dokstar_ 2019_190923.pdf

19. Swedish Association of Local Authorities and Regions (2016) Classification of Swedish municipalities 2017. Stockholm.

20. Immonen-Räihä $P$, Kauhava L, Parvinen I, Helenius H, Klemi $P$ (2001) Customer fee and participation in breast-cancer screening. Lancet 358:1425

21. Alfonzo E, Andersson Ellström A, Nemes S, Strander B (2016) Effect of fee on cervical cancer screening attendance-ScreenFee, a Swedish population-based randomised trial. PLoS ONE 11:e0150888

22. Alharbi A, Khan MM, Horner R, Brandt H, Chapman C (2019) Impact of removing cost sharing under the affordable care act (ACA) on mammography and pap test use. BMC Public Health 19:370

23. Fedewa SA, Goodman M, Flanders WD, Han X, Smith RA, E MW, Doubeni CA, Sauer AG, Jemal A (2015) Elimination of cost-sharing and receipt of screening for colorectal and breast cancer. Cancer 121:3272-3280

24. Xu WY, Wickizer TM, Jung JK (2019) Effectiveness of Medicare cost-sharing elimination for Cancer screening on utilization. BMC Health Serv Res 19:392

25. Sabatino SA, Thompson TD, Guy GP Jr, de Moor JS, Tangka FK (2016) Mammography use among medicare beneficiaries after elimination of cost sharing. Med Care 54:394-399

26. Trivedi AN, Leyva B, Lee Y, Panagiotou OA, Dahabreh IJ (2018) Elimination of cost sharing for screening mammography in medicare advantage plans. N Engl J Med 378:262-269

27. Jena AB, Huang J, Fireman B, Fung V, Gazelle S, Landrum MB, Chernew M, Newhouse JP, Hsu J (2017) Screening mammography for free: impact of eliminating cost sharing on cancer screening rates. Health Serv Res 52:191-206 
28. Lagerlund M, Hedin A, Sparén P, Thurfjell E, Lambe M (2000) Attitudes, beliefs, and knowledge as predictors of nonattendance in a Swedish population-based mammography screening program. Prev Med 31:417-428

29. Lagerlund M, Widmark C, Lambe M, Tishelman C (2001) Rationales for attending or not attending mammography screening-a focus group study among women in Sweden. Eur J Cancer Prev 10:429-442

30. Manjer ÅR, Zackrisson S, Emilsson UM (2016) On ambivalence about mammography screening: support in the decision-making process a potential role for health care social workers? Br J Soc Work 46:480-497

31. Gardner MP, Adams A, Jeffreys M (2013) Interventions to increase the uptake of mammography amongst low income women: a systematic review and meta-analysis. PLoS ONE 8:e55574

Publisher's Note Springer Nature remains neutral with regard to jurisdictional claims in published maps and institutional affiliations. 